\title{
Indirect methods for wake potential integration
}

\author{
Igor Zagorodnov \\ Deutsches Elektronen-Synchrotron (DESY), Notkestrasse 85, 22603 Hamburg, Germany
}

(Received 23 May 2006; published 16 October 2006)

\begin{abstract}
The development of modern accelerator and free-electron laser projects often requires one to consider wakefields of very short bunches in arbitrary three-dimensional structures. To obtain the wake numerically by direct integration of fields is difficult, since it can take a long time for fields scattered off the structure to catch up to the short bunch. On the other hand, no general algorithm for indirect wakefield integration exists in the literature. In this report we review the known indirect methods to compute wake potentials in rotationally symmetric and cavitylike three-dimensional structures. We present several extensions to the existing techniques and then derive a new general indirect method for arbitrary three-dimensional structures. Finally, the new method is numerically tested and applied to the problem of a rectangular collimator.
\end{abstract}

DOI: 10.1103/PhysRevSTAB.9.102002

PACS numbers: 29.27.Bd, 02.60.Cb, 02.70.Bf

\section{INTRODUCTION}

The calculation of impedances for various accelerator elements is an important part of the modern accelerator design $[1,2]$. State-of-the-art electrodynamic computer codes allow direct time-domain calculations of short-range wake potentials for the complicated geometries of real components. However, for short bunches, a long-time propagation of the electromagnetic field in the outgoing vacuum chamber is required to take into account the scattered fields which will reach the bunch at later times. To drastically reduce the computational time and avoid numerical error accumulation, several indirect integration algorithms were developed for rotationally symmetric geometries [3-7].

In Sec. II A of this paper it is shown that contrary to the statement of [7] the Napoly-Chin-Zotter (NCZ) method can be applied for the case of unequal beam tube radii. In Sec. II B we derive a modification of the NCZ method which is extended later, in Sec. III B, to three dimensions. Section III A reviews the only known three-dimensional indirect integration algorithm for cavitylike structures [8]. Finally, after generalization in Sec. IIIC of Heifets's method [9] on the situation required to calculate the transverse wake potential, we introduce in Sec. IIID a new general algorithm for a treatment of arbitrary threedimensional structures. Several numerical examples are presented to illustrate the accuracy and efficiency of the new method in Sec. IV. The new integration algorithm is numerically tested and applied to the problem of a rectangular collimator.

Finite difference numerical methods to solve the problem shown in Fig. 1 were developed in [3,4,8,10-17]. The rigid beam picture adopted in these methods is not selfconsistent, but is an excellent approximation for relativistic beams as far as the calculation of wakefields is concerned [1]. The longitudinal and the transverse wake potentials are defined as [2]

$$
\begin{gathered}
W_{\|}(\vec{r}, s)=-\frac{1}{Q} \int_{-\infty}^{\infty} E_{z}[\vec{r}, z, t(z, s)] d z, \quad \vec{r}=(x, y), \\
\vec{W}_{\perp}(\vec{r}, s)=\frac{1}{Q} \int_{-\infty}^{\infty}\left(\vec{E}_{\perp}+\vec{v} \times \vec{B}\right)[\vec{r}, z, t(z, s)] d z,
\end{gathered}
$$

where $Q$ is the total charge of the bunch, $s$ is the distance behind the given origin $z_{0}=c t$ in the exciting bunch, and $t(z, s)=(z+s) / c$.

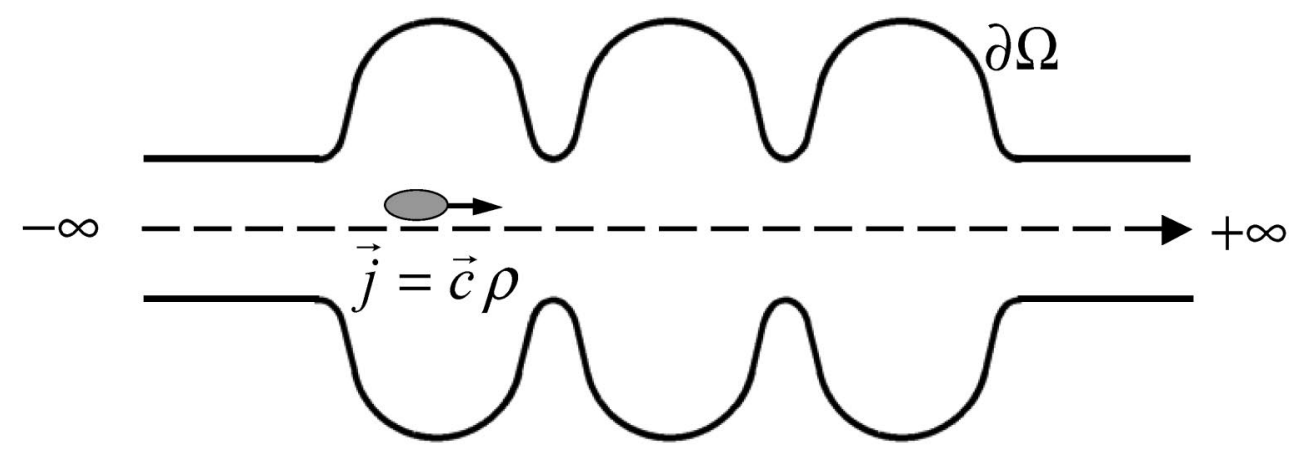

FIG. 1. Charged particle bunch moving through an accelerating structure supplied with infinite pipes. 
The purpose of this paper is to show how to replace the improper integrals in (1) and (2) by proper integrals. This is essential for computer calculations, in particular, for short bunches, where long beam tubes would require both excessive computer memory and CPU time, and tend to lead to numerical errors.

In the following, only integral (1) will be considered as the transverse potential can be derived from the longitudinal one by applying the Panofsky-Wenzel theorem [18]

$$
\frac{\partial}{\partial s} \vec{W}_{\perp}(\vec{r}, s)=\nabla_{\perp} W_{\|}(\vec{r}, s) .
$$

\section{INDIRECT METHODS FOR AXISYMMETRIC STRUCTURES}

For rotationally symmetric structures, an azimuthal Fourier expansion can be used to reduce the problem to a set of two-dimensional problems. For cavitylike structures the integration of the wakefields can be performed along a straight line parallel to the axis at the outgoing beam tube radius as was suggested by Weiland in [4] and realized in codes BCI [11], TBCI [12], and MAFIA [14]. However, this technique only works if no part of the structure extends to radii smaller than the radius of the outgoing tube. It has been realized subsequently $[5,6]$ that the potential can be calculated by integrating the wake along the perfectly conducting boundary of a structure. Finally, Napoly et al. [7] have generalized the results above by showing that the wake potentials, at all azimuthal harmonics $m$, can be expressed as integrals over the wakefields along any arbitrary contour which spans the structure longitudinally. This general method was implemented and tested in the code ABCI [13]. A modified version of this method was introduced in [15] and implemented in the code ECHO. An alternative approach based on waveguide mode expansion was introduced in [3] and realized in the code DBCI. In the following, we first review the simplest and most general method of Napoly et al. and then describe its modified version used later for the $3 \mathrm{D}$ case.

\section{A. Napoly-Chin-Zotter method for arbitrary rotational symmetric structures with unequal beam tubes radii}

In this paper we consider only structures having perfectly conducting ingoing and outgoing waveguides. The steady-state field pattern of a bunch in an ingoing perfectly conducting waveguide does not contribute to the wake potential. Hence, the improper integral for the ingoing waveguide reduces to a proper integral along a finite part of the integration path and, as will be described below, the $\mathrm{NCZ}$ method is applicable for the case where the ingoing and outgoing tubes have unequal radii (see Fig. 2).

For a bunch moving at speed of light $c$ at an offset $r_{0}$ from and parallel to the axis of a rotationally symmetric structure, the source current $\vec{j}$ can be presented as [1]

$$
\vec{j}=\frac{\vec{c} Q \lambda(z / c-t) \delta\left(r-r_{0}\right)}{\pi r_{0}} \sum_{m=0}^{\infty} \frac{\cos m \varphi}{1+\delta_{m 0}},
$$

$\int_{-\infty}^{\infty} \lambda(s) d s=1$,

where $\delta_{m 0}=1$ if $m=0,0$ if $m \neq 0$, and $\lambda(s)$ is the normalized longitudinal charge distribution, $Q$ is the bunch charge, $\delta$ is the Dirac delta function.

The scattered electromagnetic field in cylindrical coordinates $(r, \theta, z)$ can be written as

$$
\begin{aligned}
\left(E_{r}^{\mathrm{sc}}, B_{\theta}^{\mathrm{sc}}, E_{z}^{\mathrm{sc}}\right)[r, \theta, z, t(z, s)]= & \sum_{m=0}^{\infty}\left(e_{r}, b_{\theta}, e_{z}\right)^{(m)}(r, z, s) \\
& \times \cos (m \theta) \\
\left(B_{r}^{\mathrm{sc}}, E_{\theta}^{\mathrm{sc}}, B_{z}^{\mathrm{sc}}\right)[r, \theta, z, t(z, s)]= & \sum_{m=0}^{\infty}\left(b_{r}, e_{\theta}, b_{z}\right)^{(m)}(r, z, s) \\
& \times \sin (m \theta) .
\end{aligned}
$$

Substituting expansion (4) in Maxwell's equations and combining them yields [7] at each order $m$

$$
\begin{aligned}
\partial_{r}\left(r^{m}\left[e_{z}+c b_{z}\right]^{(m)}\right) & =r^{m} \partial_{z}\left[e_{r}+c b_{\theta}-e_{\theta}+c b_{r}\right]^{(m)}, \\
\partial_{r}\left(r^{-m}\left[e_{z}-c b_{z}\right]^{(m)}\right) & =r^{-m} \partial_{z}\left[e_{r}+c b_{\theta}+e_{\theta}-c b_{r}\right]^{(m)},
\end{aligned}
$$

where $\partial_{r}, \partial_{z}$ are partial derivatives.

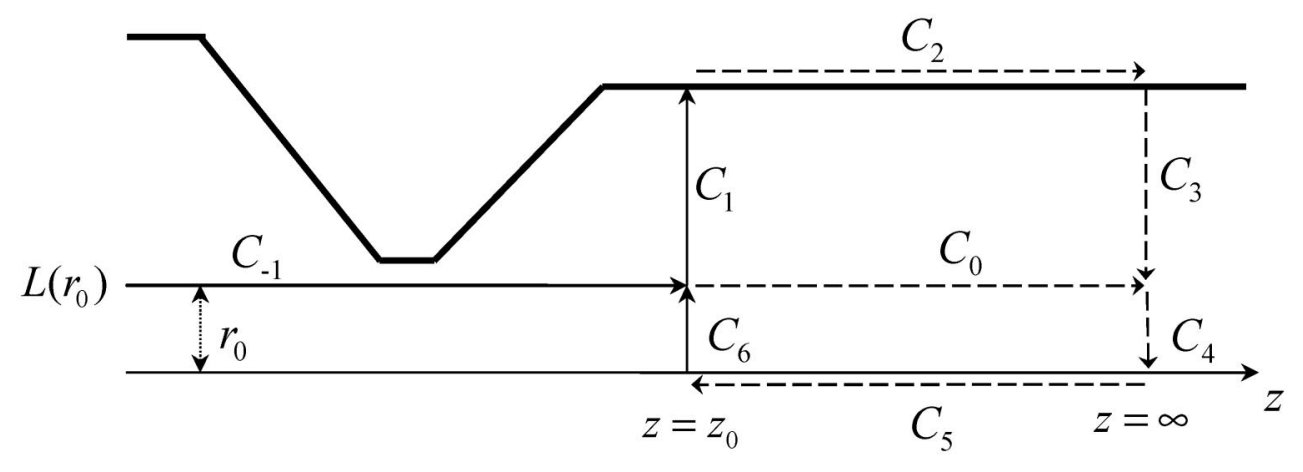

FIG. 2. Contours for the indirect integration. 
That means that the 1-forms

$$
\begin{aligned}
\omega_{S}^{(m)}= & r^{m}\left[e_{r}+c b_{\theta}-e_{\theta}+c b_{r}\right]^{(m)} d r+r^{m}\left[e_{z}+c b_{z}\right]^{(m)} d z \\
\omega_{D}^{(m)}= & r^{-m}\left[e_{r}+c b_{\theta}+e_{\theta}-c b_{r}\right]^{(m)} d r \\
& +r^{-m}\left[e_{z}-c b_{z}\right]^{(m)} d z
\end{aligned}
$$

are closed.

Recalling that the condition for 1-form $\omega=f(r, z) d r+$ $g(r, z) d z$ to be closed is

$$
d \omega=\left(\partial_{r} g-\partial_{z} f\right) d r \wedge d z=0,
$$

it follows from Stokes's theorem (symbol $\partial \Omega$ means the boundary of domain $\Omega$ )

$$
\int_{\partial \Omega} \omega=\int_{\Omega} d \omega
$$

that an integral of the closed form along a closed contour vanishes. This property allows us to deform the wakefield integration path and replace the infinite integration contour with a finite one. In the following the situation shown in Fig. 2 will be considered.

The longitudinal wake potential at mode $m$ is defined as

$$
W_{\|}^{(m)}(r, s)=-\frac{1}{Q} \int_{-\infty}^{\infty} e_{z}^{(m)}(r, z, s) d z
$$

and, for the general situation shown in Fig. 2, we can write [7]

$$
\begin{aligned}
Q W_{\|}^{(m)} & =-\int_{-\infty}^{\infty} e_{z}^{(m)} d z=-\int_{L\left(r_{0}\right)} e_{z}^{(m)} d z \\
& =-\frac{r_{0}^{m}}{2} \int_{L\left(r_{0}\right)}\left(\omega_{D}^{(m)}+a^{-2 m} \omega_{S}^{(m)}\right), \\
L\left(r_{0}\right) & =C_{-1} \cup C_{0},
\end{aligned}
$$

where $a$ denotes the pipe radius and other symbols are defined in Fig. 2. Simplifying the notation and omitting the azimuthal number, this gives

$$
\begin{aligned}
Q W_{\|}^{(m)}= & -\frac{r_{0}^{m}}{2} \int_{L\left(r_{0}\right)}\left(\omega_{D}+a^{-2 m} \omega_{S}\right) \\
= & -\frac{r_{0}^{m}}{2} \int_{C_{-1}}\left(\omega_{D}+a^{-2 m} \omega_{S}\right) \\
& +\frac{r_{0}^{m}}{2} \int_{C_{13}}\left(\omega_{D}+a^{-2 m} \omega_{S}\right), \\
C_{13}= & \bigcup_{i=1}^{3} C_{i} .
\end{aligned}
$$

For a perfectly conducting outgoing pipe the last integral becomes

$$
\begin{aligned}
\frac{r_{0}^{m}}{2} \int_{C_{13}}\left(\omega_{D}+a^{-2 m} \omega_{S}\right) & =\frac{r_{0}^{m}}{2} \int_{C_{1}}\left(\omega_{D}+a^{-2 m} \omega_{S}\right)+\frac{r_{0}^{m}}{2 a^{2 m}} \int_{C_{3}} \omega_{S} \\
& =\frac{r_{0}^{m}}{2 a^{m}} \int_{r_{0}}^{a}\left\{\left(\frac{a^{m}}{r^{m}}+\frac{r^{m}}{a^{m}}\right)\left[e_{r}+c b_{\theta}\right]+\left(\frac{a^{m}}{r^{m}}-\frac{r^{m}}{a^{m}}\right)\left[e_{\theta}-c b_{r}\right]\right\} d r+Q F^{(m)}(s)
\end{aligned}
$$

and the wake potential can be found as

$$
\begin{aligned}
W_{\|}^{(m)}= & -\frac{r_{0}^{m}}{2 Q a^{m}} \int_{C_{-1}}\left(\left[\frac{r_{0}^{m}}{a^{m}}+\frac{a^{m}}{r_{0}^{m}}\right] e_{z}-\left[\frac{r_{0}^{m}}{a^{m}}-\frac{a^{m}}{r_{0}^{m}}\right] c b_{z}\right) d z \\
& +\frac{r_{0}^{m}}{2 Q a^{m}} \int_{r_{0}}^{a}\left\{\left(\frac{a^{m}}{r^{m}}+\frac{r^{m}}{a^{m}}\right)\left[e_{r}+c b_{\theta}\right]+\left(\frac{a^{m}}{r^{m}}-\frac{r^{m}}{a^{m}}\right)\left[e_{\theta}-c b_{r}\right]\right\} d r+F^{(m)}(s),
\end{aligned}
$$

where

$$
F^{(m)}(s)= \begin{cases}\frac{\lambda(s)}{2 \pi \varepsilon_{0}} \ln \left(\frac{a}{r_{0}}\right), & m=0 \\ \frac{\lambda(s)}{2 \pi \varepsilon_{0}} \frac{r_{0}^{2 m}}{m a^{4 m}}\left(r_{0}^{2 m}-a^{2 m}\right), & m>0\end{cases}
$$

Following the NCZ method we have thus managed to replace the improper integration along the contour $C_{0}$ by the proper integral along the finite contour $C_{1}$.

\section{B. Modification of the NCZ method}

In this paragraph we introduce a modification of the NCZ method. The main feature of the modified method is that (as in the direct method) we integrate only the $e_{z}^{(m)}(r, z, s)$ component of the scattered electromagnetic field along a straight line $C_{-1}$ at radius $r_{0}$, and use other field components only at the end of the structure. This property of the method allows us to apply it for 3D calculations as described in Sec. III B.

For the general situation shown in Fig. 2 we can write 


$$
\begin{aligned}
Q W_{\|}^{(m)} & =-\int_{-\infty}^{\infty} e_{z}^{(m)} d z=-\int_{L\left(r_{0}\right)} e_{z}^{(m)} d z=-\int_{C_{-1}} e_{z}^{(m)} d z-\int_{C_{0}} e_{z}^{(m)} d z, \quad L\left(r_{0}\right)=C_{-1} \cup C_{0}, \\
-\int_{C_{0}} e_{z}^{(m)} d z & =-\frac{1}{2}\left(\int_{C_{0}}\left(r_{0}^{m} \omega_{D}^{(m)}+r_{0}^{-m} \omega_{S}^{(m)}\right)+\frac{\beta}{a^{m}} \int_{C_{16}} \omega_{S}^{(m)}\right)=\frac{1}{2}\left(\int_{C_{13}}\left(r_{0}^{m} \omega_{D}^{(m)}+r_{0}^{-m} \omega_{S}^{(m)}\right)-\frac{\beta}{a^{m}} \int_{C_{16}} \omega_{S}^{(m)}\right), \\
\beta & =\left(\frac{a}{r_{0}}\right)^{m}-\left(\frac{a}{r_{0}}\right)^{-m}, \quad C_{16}=\bigcup_{i=1}^{6} C_{i} .
\end{aligned}
$$

For a perfectly conducting geometry this last equation reduces to

$$
\int_{C_{0}} e_{z}^{(m)} d z=-\frac{1}{2}\left[\int_{C_{1}}\left(r_{0}^{m} \omega_{D}^{(m)}+r_{0}^{-m} \omega_{S}^{(m)}-\frac{\beta}{a^{m}} \omega_{S}^{(m)}\right)-\frac{\beta}{a^{m}} \int_{C_{6}} \omega_{S}^{(m)}\right],
$$

and the wake potential can be found as

$$
\begin{aligned}
Q W_{\|}^{(m)}= & -\int_{C_{-1}} e_{z}^{(m)} d z+\frac{\beta}{2 a^{m}} \int_{0}^{a} r^{m}\left[e_{r}+c b_{\theta}-e_{\theta}+c b_{r}\right]^{(m)} d r \\
& -\frac{1}{2} \int_{r_{0}}^{a}\left\{\left(\frac{r_{0}^{m}}{r^{m}}+\frac{r_{0}^{-m}}{r^{-m}}\right)\left[e_{r}+c b_{\theta}\right]^{(m)}+\left(\frac{r_{0}^{m}}{r^{m}}-\frac{r_{0}^{-m}}{r^{-m}}\right)\left[e_{\theta}-c b_{r}\right]^{(m)}\right\} d r .
\end{aligned}
$$

Again we have managed to replace the improper integration along the contour $C_{0}$ by proper integrals along the finite contours $C_{1}, C_{6}$.

\section{INDIRECT METHODS FOR 3D STRUCTURES}

In the previous chapter a general solution for rotationally symmetric geometries was described. However, the NCZ method does not generalize easily to three dimensions and so we look here for alternative techniques. In Sec. III B we generalize the NCZ method to the case of general 3D structures with outgoing round pipe. In Sec. III C we extend Heifets's method [9] to the situation required to calculate the transverse wake potential. Finally, in Sec. IIID we introduce a new general algorithm for a treatment of arbitrary three-dimensional structures.

\section{A. Method for cavitylike structures or structures with small outgoing waveguide}

In a similar manner to the rotationally symmetric case, the integration through a waveguide gap results in a simple and efficient algorithm [8].

As shown in [8], the longitudinal wake potential is a harmonic function of the transverse coordinates

$$
\Delta_{\perp} W_{\|}(\vec{r}, s)=0, \quad \vec{r}=(x, y) \in \Omega_{\max }^{\perp},
$$

where $\Omega_{\max }^{\perp}$ is the transverse area defined by the intersection of all transverse cross sections (see, for example, Fig. 3) and $\Delta_{\perp}$ is the two-dimensional Laplace operator. Hence, for cavitylike structures the relation $\Omega_{\text {out }}^{\perp}=\Omega_{\max }^{\perp}$ holds and we perform the integration at the transverse position of the outgoing waveguide boundary $\partial \Omega_{\text {out }}^{\perp}$. The longitudinal wake potential for any position inside the waveguide is then obtained as a solution of Laplace's equation (7) with the Dirichlet boundary condition

$$
W_{\|}(\vec{r}, s)=W_{\|}^{\operatorname{direct}}(\vec{r}, s), \quad \vec{r}=(x, y) \in \partial \Omega_{\text {out }}^{\perp} .
$$

However, this method does not work if the area $\Omega_{\max }^{\perp}$ is smaller than the outgoing waveguide intersection $\Omega_{\text {out }}^{\perp}$. In the following, we suggest methods which are appropriate for just such a situation.

\section{B. Method for general 3D structures with outgoing round pipe}

In this paragraph we consider the situation where an arbitrary three-dimensional structure is supplied with a round outgoing pipe. In this case we can easily generalize the method of Sec. II B as follows.
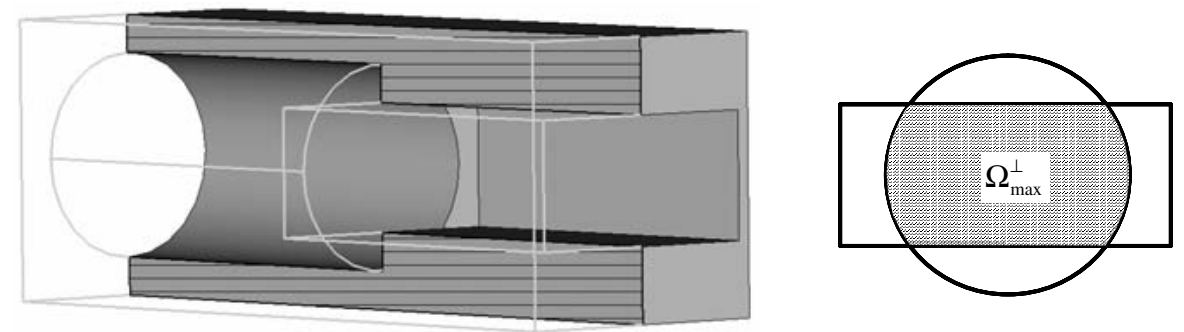

FIG. 3. Round to rectangular transition and the maximal area for wake potential calculation. 


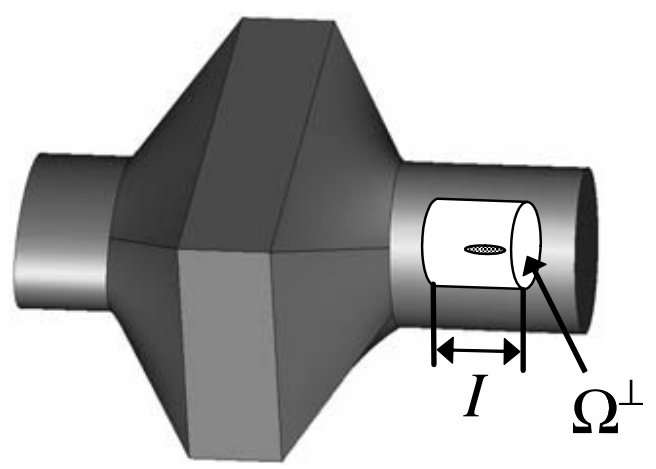

FIG. 4. The tapered cavity and area $\Omega^{\perp} \times I$ around the bunch.

Let us suggest that we are interested in the wake potential in area $\Omega^{\perp} \times I$ around the bunch as shown in Fig. 4 .

As in the rotationally symmetric case, let us take the integration path $L\left(\vec{r}_{0}\right)$ as the union of the path $C_{-1}\left(\vec{r}_{0}\right)$ through the 3D structure up to the point $z_{0}$ and the path $C_{0}\left(\vec{r}_{0}\right)$ completely inside of the outgoing round pipe:

$$
L\left(\vec{r}_{0}\right)=C_{-1}\left(\vec{r}_{0}\right) \cup C_{0}\left(\vec{r}_{0}\right), \quad \vec{r}_{0} \in \Omega^{\perp} .
$$

The wake potential can be written as

$$
\begin{aligned}
Q W_{\|}\left(\vec{r}_{0}, s\right)= & -\int_{C_{-1}\left(\vec{r}_{0}\right)} E_{z}^{\mathrm{sc}}\left[\vec{r}_{0}, z, t(z, s)\right] d z \\
& -\int_{C_{0}\left(\vec{r}_{0}\right)} E_{z}^{\mathrm{sc}}\left[\vec{r}_{0}, z, t(z, s)\right] d z, \quad \vec{r}_{0} \in \Omega^{\perp} .
\end{aligned}
$$

Our purpose is to replace the second improper integral by proper integrals. This can be achieved by straightforward generalization of the method described in Sec. II B.

Indeed, after the bunch has arrived in the round pipe, we can use an azimuthal Fourier expansion to reduce the 3D problem to a set of $2 \mathrm{D}$ problems. However, in contrast to the rotationally symmetric case of Sec. II, the electromagnetic field components are now complex quantities due to the fields scattered before by the 3D structure.

Let us represent the scattered electromagnetic field $\vec{F}=$ $\left(\vec{E}^{\mathrm{sc}}, \vec{B}^{\mathrm{sc}}\right)$ as

$$
\begin{aligned}
\vec{F}(z, r, \theta) & =\operatorname{Re} \sum_{m=0}^{\infty} \vec{F}_{m}(z, r) e^{-i m \theta} \\
& =\operatorname{Re} \sum_{m=0}^{\infty}\left[\vec{F}_{m}^{\operatorname{Re}}(z, r)+i \vec{F}_{m}^{\operatorname{Im}}(z, r)\right] e^{-i m \theta}, \\
\vec{F}_{m}(z, r) & =2 \int_{0}^{2 \pi} \vec{F}(z, r, \theta) e^{i m \theta} \frac{d \theta}{2 \pi}, \\
\vec{F}_{0}(z, r) & =\int_{0}^{2 \pi} \vec{F}(z, r, \theta) \frac{d \theta}{2 \pi} .
\end{aligned}
$$

Then the equations for the azimuthal harmonics at each number $m$ separate into two independent sets, each of which can be written in the form (we simplify the notation and omit the azimuthal number)

$$
\begin{aligned}
\frac{1}{r} \frac{\partial}{\partial r}\left(r b_{r}\right)+\frac{M}{r} b_{\theta}+\frac{\partial}{\partial z} b_{z} & =0, \\
\frac{1}{r} \frac{\partial}{\partial r}\left(r e_{r}\right)+\frac{M}{r} e_{\theta}+\frac{\partial}{\partial z} e_{z} & =0, \\
\frac{M}{r} b_{z}-\frac{\partial}{\partial z} b_{\theta} & =\frac{\partial}{c^{2} \partial t} e_{r}, \\
-\frac{M}{r} e_{z}-\frac{\partial}{\partial z} e_{\theta} & =-\frac{\partial}{\partial t} b_{r}, \\
\frac{\partial}{\partial z} b_{r}-\frac{\partial}{\partial r} b_{z} & =\frac{\partial}{c^{2} \partial t} e_{\theta}, \\
\frac{\partial}{\partial z} e_{r}-\frac{\partial}{\partial r} e_{z} & =-\frac{\partial}{\partial t} b_{\theta}, \\
\frac{1}{r} \frac{\partial}{\partial r}\left(r b_{\theta}\right)-\frac{M}{r} b_{r} & =\frac{\partial}{c^{2} \partial t} e_{z}, \\
\frac{1}{r} \frac{\partial}{\partial r}\left(r e_{\theta}\right)+\frac{M}{r} e_{r} & =-\frac{\partial}{\partial t} b_{z},
\end{aligned}
$$

where $M=m$ for $e_{z}^{\mathrm{Re}}, e_{r}^{\mathrm{Re}}, b_{\theta}^{\mathrm{Re}}, b_{z}^{\mathrm{Im}}, b_{r}^{\mathrm{Im}}, e_{\theta}^{\mathrm{Im}}$ and $M=-m$ for $e_{z}^{\mathrm{Im}}, e_{r}^{\mathrm{Im}}, b_{\theta}^{\mathrm{Im}}, b_{z}^{\mathrm{Re}}, b_{r}^{\mathrm{Re}}, e_{\theta}^{\mathrm{Re}}$.

Following the approach in Sec. II B, we can show that the following relations hold:

$$
\begin{aligned}
\int_{C_{0}} e_{z}^{\mathrm{Re},(m)} d z= & -\frac{1}{2}\left[\int_{C_{1}}\left(r_{0}^{m} \omega_{D}^{(m)}+r_{0}^{-m} \omega_{S}^{(m)}-\frac{\beta}{a^{m}} \omega_{S}^{(m)}\right)\right. \\
& \left.-\frac{\beta}{a^{m}} \int_{C_{6}} \omega_{S}^{(m)}\right], \\
\int_{C_{0}} e_{z}^{\operatorname{Im},(m)} d z= & -\frac{1}{2}\left[\int _ { C _ { 1 } } \left(r_{0}^{m} \omega_{S}^{(-m)}+r_{0}^{-m} \omega_{D}^{(-m)}\right.\right. \\
& \left.\left.-\frac{\beta}{a^{m}} \omega_{D}^{(-m)}\right)-\frac{\beta}{a^{m}} \int_{C_{6}} \omega_{D}^{(-m)}\right] .
\end{aligned}
$$

The second integral in relation (8) can be written as

$$
\begin{aligned}
\int_{C_{0}\left(\vec{r}_{0}\right)} E_{z}^{\mathrm{sc}}\left(\vec{r}_{0}, z, s\right) d z= & \sum_{m=0}^{\infty}\left[\int_{C_{0}} e_{z}^{\mathrm{Re},(m)} d z \cos \left(m \theta_{0}\right)\right. \\
& \left.+\int_{C_{0}} e_{z}^{\mathrm{Im},(m)} d z \sin \left(m \theta_{0}\right)\right] \\
\vec{r}_{0}= & \left(r_{0}, \theta_{0}\right) \in \Omega^{\perp},
\end{aligned}
$$

and substitution of Eqs. (9) reduces this improper integral along the $z$-axis to a sum of proper integrals along the radius.

\section{Method based on the directional symmetry of wake potential}

The methods introduced in the previous sections are not completely general. The method of Sec. III A allows us to treat only structures where the cross section of the outgoing waveguide is covered by any other cross section along the 
structure. For example, if we are interested in the wake for the transition from a round pipe to a rectangular one, as shown in Fig. 3, then this method does not work. The method of Sec. III B is not applicable directly either.

However, often we are able to apply one of the two methods when the bunch direction of motion is reversed. For example, the inverse transition from a rectangular to a round pipe can be treated with the algorithm of Sec. III B.

In this section we describe a method which allows us to calculate the wake potential for one direction from the wake potential for the reversed one.

In [9], a directional symmetry of the impedance

$$
Z_{\|}(\omega, \vec{r})=\int_{-\infty}^{\infty} W_{\|}(\tau, \vec{r}) e^{-i \omega \tau} d \tau, \quad \vec{r}=(x, y),
$$

was considered and the relation between the forward impedance $Z^{+}\left(\omega, \vec{r}_{e}\right)$ and the "reversed" impedance $Z^{-}\left(\omega, \vec{r}_{e}\right)$ at the exciting charge position $\vec{r}_{e}$ was found:

$$
\begin{aligned}
Z^{-}\left(\omega, \vec{r}_{e}\right)-Z^{+}\left(\omega, \vec{r}_{e}\right)= & \frac{2}{Q^{2}} \operatorname{Re}\left(\int_{\Omega_{\text {in }}^{\perp}} \vec{E}^{+} \times \vec{H}^{-} d \vec{\mu}(\vec{r})\right. \\
& \left.-\int_{\Omega_{\text {out }}^{\perp}} \vec{E}^{+} \times \vec{H}^{-} d \vec{\mu}(\vec{r})\right),
\end{aligned}
$$

where $\Omega_{\text {in }}^{\perp}$ denotes the ingoing and $\Omega_{\text {out }}^{\perp}$ is the outgoing pipe cross section. However, in order to apply the Panofsky-Wenzel theorem and find the transverse wake potential, we need to know the longitudinal wake potential not only at the position of the exciting point charge $\vec{r}_{e}$ but in the vicinity of it.

In the following we generalize Eq. (10) in order to also be able to calculate the transverse wake potential. Let us consider a perfectly conducting structure traversed by two point charges traveling parallel to the $z$-axis in opposite directions and with offsets $\vec{r}_{1}$ and $\vec{r}_{2}$, respectively. The current densities in frequency domain are

$$
\begin{gathered}
J_{z}^{+}(\vec{r}, z)=Q \delta\left(\vec{r}-\vec{r}_{1}\right) e^{-i k z}, \quad k=\omega / c, \\
J_{z}^{-}(\vec{r}, z)=-Q \delta\left(\vec{r}-\vec{r}_{2}\right) e^{i k z},
\end{gathered}
$$

where $Q$ is the charge.

From the Lorentz reciprocity theorem [19], we obtain

$$
\begin{aligned}
Z^{-}\left(\omega, \vec{r}_{2}, \vec{r}_{1}\right)-Z^{+}\left(\omega, \vec{r}_{1}, \vec{r}_{2}\right) & =\frac{1}{Q^{2}}\left(\int_{\Omega_{\text {in }}^{\perp}} \vec{F}\left(\vec{r}_{1}, \vec{r}_{2}, \vec{r}\right) d \vec{\mu}(\vec{r})-\int_{\Omega_{\text {out }}^{\perp}} \vec{F}\left(\vec{r}_{1}, \vec{r}_{2}, \vec{r}\right) d \vec{\mu}(\vec{r})\right), \\
\vec{F}\left(\vec{r}_{1}, \vec{r}_{2}, \vec{r}\right) & =\vec{E}^{+}\left(\vec{r}_{1}, \vec{r}\right) \times \vec{H}^{-}\left(\vec{r}_{2}, \vec{r}\right)-\vec{E}^{-}\left(\vec{r}_{2}, \vec{r}\right) \times \vec{H}^{+}\left(\vec{r}_{1}, \vec{r}\right) .
\end{aligned}
$$

In order to calculate the right-hand side, we note that at infinity the field pattern of the charge can be found by solving the two-dimensional Poisson equation:

$$
\begin{aligned}
& \Delta \varphi_{i}(\vec{r})=Z_{0} Q \delta\left(\vec{r}-\vec{r}_{i}\right), \quad \vec{r} \in\left\{\Omega_{\text {in }}^{\perp}, \Omega_{\text {out }}^{\perp},\right. \\
& \varphi_{i}(\vec{r})=0, \quad \vec{r} \in\left\{\partial \Omega_{\text {in }}^{\perp}, \partial \Omega_{\text {out }}^{\perp}, \quad i=1,2,\right.
\end{aligned}
$$

where $Z_{0}$ is the impedance of free space.

To show this observe that electric fields at infinity can be written as

$$
\begin{aligned}
& \vec{E}_{+}\left(\vec{r}_{1}, \vec{r}, z\right)=e^{-i k z} \vec{E}^{+}\left(\vec{r}_{1}, \vec{r}\right)=e^{-i k z} \nabla \varphi_{1}(\vec{r}), \\
& \vec{E}_{-}\left(\vec{r}_{2}, \vec{r}, z\right)=e^{i k z} \vec{E}^{-}\left(\vec{r}_{2}, \vec{r}\right)=e^{i k z} \nabla \varphi_{2}(\vec{r}) .
\end{aligned}
$$

Substituting this representation into Maxwell's equation,

$$
\begin{aligned}
\nabla \times \nabla \times \vec{E}_{ \pm} & =(\omega / c)^{2} \vec{E}_{ \pm}-i \omega \mu \vec{J}^{ \pm}, \\
\vec{J}^{ \pm} & =\left(0,0, J_{z}^{ \pm}\right),
\end{aligned}
$$

yields Eq. (12). Additionally, from representations (13) and Maxwell's equation

$$
\nabla \times \vec{E}_{ \pm}=-i \omega \mu \vec{H}_{ \pm},
$$

we obtain that at infinity the following relations hold:

$$
\vec{H}^{+}=\frac{1}{Z_{0}} \vec{e}_{z} \times \vec{E}^{+}, \quad \vec{H}^{-}=-\frac{1}{Z_{0}} \vec{e}_{z} \times \vec{E}^{-} .
$$

Hence, Eq. (11) can be written as

$$
\begin{aligned}
Z^{-}\left(\omega, \vec{r}_{2}, \vec{r}_{1}\right)-Z^{+}\left(\omega, \vec{r}_{1}, \vec{r}_{2}\right)=2 Z^{e}\left(\vec{r}_{1}, \vec{r}_{2}\right), & \\
Z^{e}\left(\vec{r}_{1}, \vec{r}_{2}\right)= & \frac{1}{Q^{2} Z_{0}}\left(\int_{\Omega_{\text {in }}^{\perp}}\left(\nabla \varphi_{1}, \nabla \varphi_{2}\right) d \mu(\vec{r})\right. \\
& \left.-\int_{\Omega_{\text {out }}^{\perp}}\left(\nabla \varphi_{1}, \nabla \varphi_{2}\right) d \mu(\vec{r})\right)
\end{aligned}
$$

and the forward and reverse wake potentials are related according to

$$
\begin{gathered}
W^{-}\left(s, \vec{r}_{2}, \vec{r}_{1}\right)-W^{+}\left(s, \vec{r}_{1}, \vec{r}_{2}\right)=2 W^{e}\left(s, \vec{r}_{1}, \vec{r}_{2}\right), \\
W^{e}\left(s, \vec{r}_{1}, \vec{r}_{2}\right)=\int_{-\infty}^{s} w_{\delta}^{e}\left(s-s^{\prime}\right) \lambda\left(s^{\prime}\right) d s^{\prime}=c Z^{e}\left(\vec{r}_{1}, \vec{r}_{2}\right) \lambda(s), \\
w_{\delta}^{e}(s)=\int_{-\infty}^{\infty} Z^{e}\left(\vec{r}_{1}, \vec{r}_{2}\right) e^{i(\omega / c) s} \frac{d \omega}{2 \pi}=c \delta(s) Z^{e}\left(\vec{r}_{1}, \vec{r}_{2}\right) .
\end{gathered}
$$


Hence, in order to find the wake potential of the round to rectangular transition we can calculate the wake potential of the inverse transition with the method of Sec. III B. Then we need to calculate fields (13) in both pipe cross sections. This is a two-dimensional problem and can be solved either analytically or numerically. Finally, we use Eq. (14) to obtain the required wake potential. The numerical application of this method for the calculation of the wakes can be found in [20].

\section{General method for arbitrary 3D geometries}

In this section we present a new general method for arbitrary $3 \mathrm{D}$ geometries. The method is derived through a waveguide mode expansion and is sketched in Fig. 5. In the following we will show how to replace the direct integration along infinite contour $C_{0}$ by a solution of mathematical problems in waveguide cross section $\Omega_{\text {out }}^{\perp}$.

The longitudinal component $E_{z}$ of the scattered electric field in the outgoing waveguide can be written as a linear combination of the $z$-components of the TM waveguide modes [19],

$$
\begin{gathered}
E_{z}^{\mathrm{sc}}(\vec{r}, z, t)=\sum_{n} E_{n}(\vec{r}) g_{n}(z, t), \quad \vec{r}=(x, y) \in \Omega_{\mathrm{out}}^{\perp}, \\
g_{n}(z, t)=\int_{-\infty}^{\infty} \alpha_{n}(\omega) e^{i\left(\beta_{n} z-\omega t\right)} d \omega,
\end{gathered}
$$

where $\beta_{n}$ is a propagation factor associated with mode $E_{n}(\vec{r})$.

In the general case, we can again represent the wake potential as sum of two integrals

$$
\begin{aligned}
Q W_{\|}\left(\vec{r}_{0}, s\right)= & -\int_{C_{-1}\left(\vec{r}_{0}, z_{0}-s\right)} E_{z}^{\mathrm{sc}}\left[\vec{r}_{0}, z, t(z, s)\right] d z \\
& -\int_{C_{0}\left(\vec{r}_{0}, z_{0}-s\right)} E_{z}^{\mathrm{sc}}\left[\vec{r}_{0}, z, t(z, s)\right] d z \\
t(z, s)= & \frac{z+s}{c},
\end{aligned}
$$

where $z_{0}$ is located inside of outgoing waveguide as shown in Fig. 5.
The second integral can be written as

$$
\begin{aligned}
& \int_{C_{0}\left(\vec{r}_{0}, z_{0}-s\right)} E_{z}^{\mathrm{sc}}\left[\vec{r}_{0}, z, t(z, s)\right] d z \\
& \quad=\int_{z_{0}-s}^{\infty} \sum_{n} E_{n}\left(\vec{r}_{0}\right) \int_{-\infty}^{\infty} \alpha_{n}(\omega) e^{i\left[\beta_{n} z-\omega(z+s) / c\right]} d \omega d z,
\end{aligned}
$$

where we have used Eq. (15). Changing the integration and the summation we obtain

$$
\begin{aligned}
& \int_{C_{0}\left(\vec{r}_{0}, z_{0}-s\right)} E_{z}^{\mathrm{sc}}\left[\vec{r}_{0}, z, t(z, s)\right] d z \\
& =\sum_{n} E_{n}\left(\vec{r}_{0}\right) \int_{-\infty}^{\infty} \alpha_{n}(\omega) \int_{z_{0}-s}^{\infty} e^{i\left[\beta_{n} z-\omega(z+s) / c\right]} d z d \omega \\
& =-\sum_{n} E_{n}\left(\vec{r}_{0}\right) \int_{-\infty}^{\infty} \alpha_{n}(\omega) \frac{e^{i\left[\beta_{n}\left(z_{0}-s\right)-\omega\left(z_{0} / c\right)\right]}}{i\left(\beta_{n}-\frac{\omega}{c}\right)} d \omega .
\end{aligned}
$$

Finally, we can write

$$
\begin{aligned}
& \sum_{n} E_{n}\left(\vec{r}_{0}\right) \int_{-\infty}^{\infty} \alpha_{n}(\omega) \frac{e^{i\left[\beta_{n}\left(z_{0}-s\right)-\omega\left(z_{0} / c\right)\right]}}{i\left(\beta_{n}-\frac{\omega}{c}\right)} d \omega \\
& =\sum_{n} E_{n}\left(\vec{r}_{0}\right) \int_{-\infty}^{\infty} \alpha_{n}(\omega) e^{i\left[\beta_{n}\left(z_{0}-s\right)-\omega\left(z_{0} / c\right)\right]} i \frac{\left(\beta_{n}+\omega / c\right)}{\beta_{n}^{2}-\omega^{2} c^{-2}} d \omega \\
& =-\sum_{n} \frac{E_{n}\left(\vec{r}_{0}\right)}{k_{n}^{2}} \int_{-\infty}^{\infty} \alpha_{n}(\omega) e^{i\left[\beta_{n}\left(z_{0}-s\right)-\omega\left(z_{0} / c\right)\right]} i\left(\beta_{n}+\frac{\omega}{c}\right) d \omega,
\end{aligned}
$$

where $k_{n}^{2}=\left(\omega c^{-1}\right)^{2}-\beta_{n}^{2}$ is a squared cutoff wave number for mode $n$ [19].

From the last expression and Eq. (16), we obtain

$$
\begin{aligned}
\int_{z_{0}-s}^{\infty} E_{z}^{\mathrm{sc}}\left[\vec{r}_{0}, z, t(z, s)\right] d z= & \sum_{n} \frac{E_{n}\left(\vec{r}_{0}\right)}{k_{n}^{2}}\left[\frac{\partial}{\partial s}+\frac{\partial}{c \partial t}\right] \\
& \times g_{n}\left(z_{0}-s, t_{0}\right),
\end{aligned}
$$

where $t_{0}=z_{0} c^{-1}$ and

$$
g_{n}\left(z_{0}-s, t_{0}\right)=\int_{\Omega_{\text {out }}^{\perp}} E_{z}^{\text {sc }}\left(x, y, z_{0}-s, t_{0}\right) E_{n}(x, y) d x d y .
$$

Equation (18) represents the main result of this paper. It reduces the improper integral along the $z$-axis to the sum of
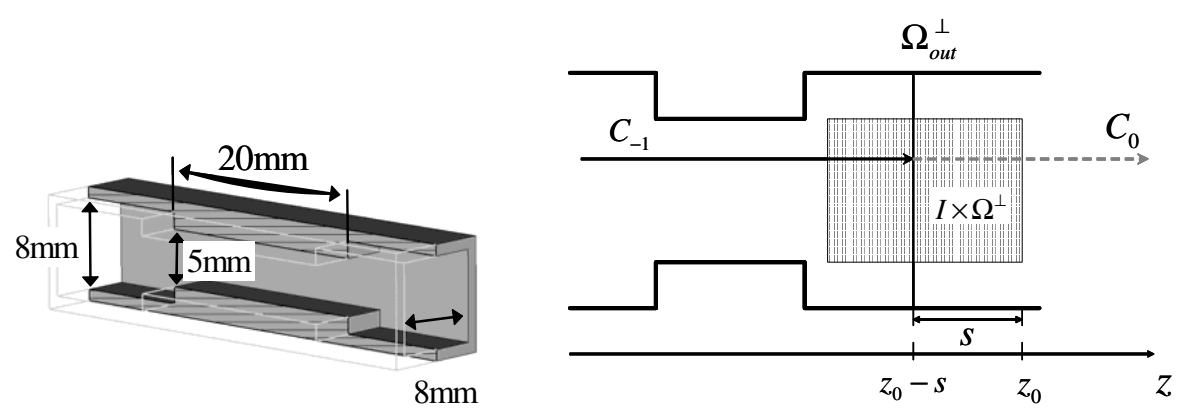

FIG. 5. The rectangular collimator. Contour $C_{-1}$ for direct integration and cross section $\Omega_{\text {out }}^{\perp}$ for indirect integration algorithm. 
proper integrals (19) in the transverse waveguide cross section $\Omega_{\text {out }}^{\perp}$.

Let us now describe a numerical algorithm. As a first step we should find the eigenmodes $\left\{E_{n}(\vec{r})\right\}$ and the eigenvalues $\left\{k_{n}^{2}\right\}$ from the solution of the eigenvalue problem [19]:

$$
\Delta E_{n}(\vec{r})=-k_{n}^{2} E_{n}(\vec{r}), \quad \vec{r}=(x, y) \in \Omega_{\text {out }}^{\perp}
$$

Next we have to find coefficient functions (19) at two instants of time: $t_{0}-0.5 \Delta t$ and $t_{0}+0.5 \Delta t$. Finally, we approximate Eq. (18) as

$$
\begin{aligned}
\int_{z_{0}-s}^{\infty} E_{z}^{\mathrm{sc}}\left(\vec{r}_{0}, z, t=\frac{z+s}{c}\right) d z & =\sum_{n} \frac{E_{n}\left(\vec{r}_{0}\right)}{k_{n}^{2}}\left[\frac{\partial}{\partial s}+\frac{\partial}{c \partial t}\right] g_{n}\left(z_{0}-s, t_{0}\right) \\
& =\sum_{n} \frac{E_{n}\left(\vec{r}_{0}\right)}{k_{n}^{2}}\left[\frac{g_{n}\left(z_{0}-s+0.5 \Delta s, t_{0}+0.5 \Delta t\right)-g_{n}\left(z_{0}-s-0.5 \Delta s, t_{0}-0.5 \Delta t\right)}{\Delta s}\right]+O\left(\Delta s^{2}\right)
\end{aligned}
$$

where $\Delta s=c \Delta t$.

Note, that problem (20) is a two-dimensional one and can be solved by an eigenvalue solver for Laplace's equation.

The algorithm based on Eq. (18) has been implemented in the three-dimensional version of the code ECHO [16] and used for calculations presented in [21,22].

After submission of this paper, we have realized that the sum in the right-hand side of Eq. (18) constitutes an eigenmode expansion of the solution $u$ of Poisson's equation:

$$
\begin{aligned}
\Delta u(\vec{r}, s) & =-\left[\frac{\partial}{\partial s}+\frac{\partial}{c \partial t}\right] E_{z}^{\mathrm{sc}}\left(\vec{r}, z_{0}-s, t_{0}\right), \quad \vec{r} \in \Omega_{\text {out }}^{\perp}, \\
u(\vec{r}, s) & =0, \quad \vec{r} \in \partial \Omega_{\text {out }}^{\perp}
\end{aligned}
$$

Indeed, if we write

$$
u(\vec{r}, s)=\sum_{n} \frac{E_{n}(\vec{r})}{k_{n}^{2}}\left[\frac{\partial}{\partial s}+\frac{\partial}{c \partial t}\right] g_{n}\left(z_{0}-s, t_{0}\right)
$$

and apply Laplace's operator to this equation, we will obtain Eq. (21). Hence, the longitudinal wake potential can be written as

$$
\begin{aligned}
Q W_{\|}\left(\vec{r}_{0}, s\right) & =-\int_{C_{-1}\left(\vec{r}_{0}, z_{0}-s\right)} E_{z}^{\mathrm{sc}}\left[\vec{r}_{0}, z, t(z, s)\right] d z-u\left(\vec{r}_{0}, s\right), \\
t(z, s) & =\frac{z+s}{c} .
\end{aligned}
$$

Above we derived an algorithm where the right-hand side of Eq. (21) is required at the time $t_{0}$ but for different positions $z=z_{0}-s$ along the structure. Alternatively, we can derive another representation

$$
\begin{aligned}
Q W_{\|}\left(\vec{r}_{0}, s\right) & =-\int_{C_{-1}\left(\vec{r}_{0}, z_{0}\right)} E_{z}^{\mathrm{sc}}\left(\vec{r}_{0}, z, t(z, s)\right) d z-w\left(\vec{r}_{0}, s\right), \\
\Delta w(\vec{r}, s) & =\left[\frac{\partial}{\partial z}-\frac{\partial}{c \partial t}\right] E_{z}^{\mathrm{sc}}\left(\vec{r}, z_{0}, t=t_{0}+s / c\right), \quad \vec{r} \in \Omega_{\text {out }}^{\perp}, \\
w(\vec{r}, s) & =0, \quad \vec{r} \in \partial \Omega_{\text {out }}^{\perp},
\end{aligned}
$$

where the right-hand side of Eq. (22) is required at the same position $z_{0}$, but for different times $t=t_{0}+s / c$.
Equation (22) has been obtained with an alternative technique by Henke and Bruns [23].

\section{NUMERICAL EXAMPLES}

In this section we present several numerical tests which confirm the accuracy and high efficiency of the suggested indirect methods for wake potential integration.

The wakes of the round-to-rectangular transition shown in Fig. 3 are calculated by the methods of Secs. III B and III $\mathrm{C}$ in Ref. [20]. Hence, we consider here only numerical tests for the most general indirect method described in Sec. III D.

As the first example, we consider the round stepped collimator shown in Fig. 6 with dimensions $a=8 \mathrm{~mm}$, $b=5 \mathrm{~mm}$, and $c=20 \mathrm{~mm}$. The longitudinal wake potential for a Gaussian bunch moving along the axis with the rms length $\sigma=20 \mu \mathrm{m}$ is shown on the left-hand side of Fig. 7. We compare the wake potentials calculated by the direct method [see Eq. (1)] against the wake potential calculated by the indirect method of Sec. II B. The wake potentials are obtained by direct integration of the longitudinal electric field component $E_{z}$ at the radius $r=$ $2.5 \mathrm{~mm}$ along the $z$-axis for different distances between 0.25 and $4 \mathrm{~m}$. This numerical check shows that the catch-up distance is more than $4 \mathrm{~m}$. The above numerical results are obtained with the code ECHO in a rotationally symmetric geometry [15]. In order to check the implementation of the 3D indirect method of Sec. III D, we have calculated the same example with the 3D version of the code ECHO [17]. For 3D calculations we used the same longitudinal mesh

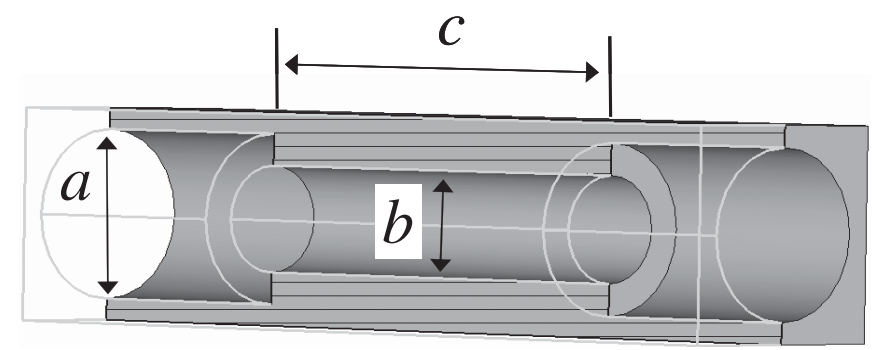

FIG. 6. The round stepped collimator. 

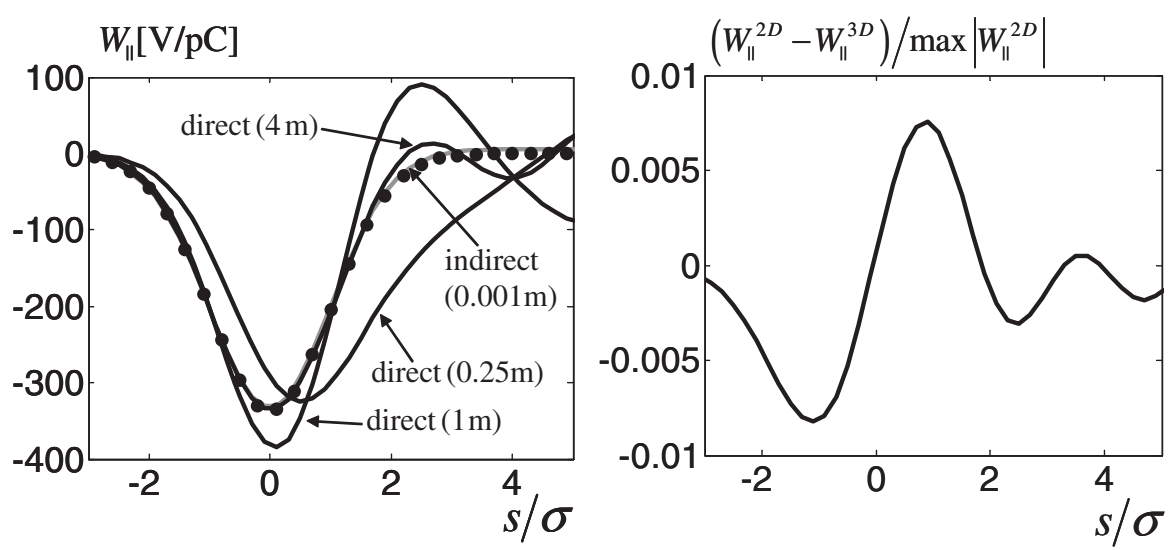

FIG. 7. Wake potentials for the round stepped collimator calculated with direct and indirect methods. Comparison of indirect wake potentials calculated with 2D (Sec. II B) and 3D (Sec. III D) methods.

step as for the $2 \mathrm{D}$ code. The comparison of $2 \mathrm{D}$ and $3 \mathrm{D}$ results is shown on the right-hand side of Fig. 7. Additionally, we have found that the numerical results agree well with the analytical approximation for the stepped collimator [2]. This analytical result is shown by circles in Fig. 7.

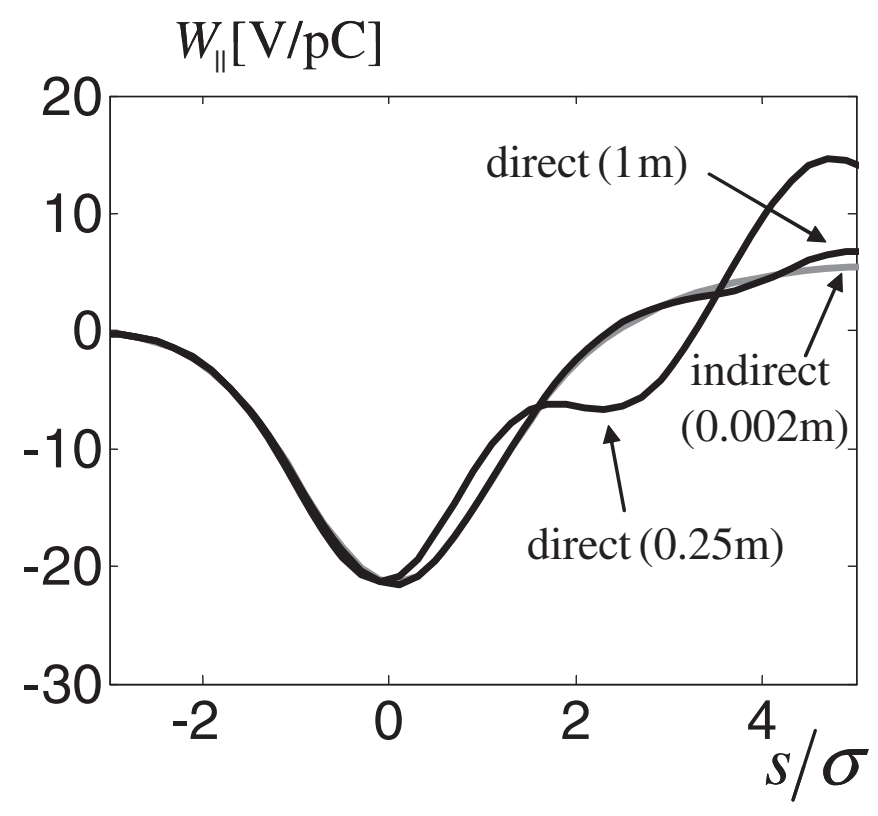

FIG. 8. Wake potential of rectangular collimator calculated with direct and indirect (Sec. III D) methods.

TABLE I. Comparison of CPU times for the example of the rectangular collimator.

\begin{tabular}{lc}
\hline \hline \multicolumn{1}{c}{ Method } & CPU time $(\mathrm{sec})$ \\
\hline Indirect, Eq. (18) & 5 \\
Indirect, Eq. (21) & 51 \\
Direct (along 1 m) & $1.5 \times 10^{4}$ \\
\hline \hline
\end{tabular}

As the next example, we consider a rectangular collimator shown in Fig. 5. Figure 8 compares the wake potentials for a Gaussian bunch moving along the axis with the rms length $\sigma=200 \mu \mathrm{m}$, calculated by the direct method, and the wake potential calculated by the indirect method of Sec. III D. The wake potentials are obtained with direct integration up to distances 0.25 and $1 \mathrm{~m}$ after the collimator. We again see that the indirect method applied at $z_{0}=$ $2 \mathrm{~mm}$ after the collimator yields an accurate result, which agrees with the direct calculation up to $1 \mathrm{~m}$.

The calculation time for the indirect integration algorithm is comparable with one integration step of the timedomain numerical scheme for Maxwell's equations. The direct integration in an outgoing waveguide requires not only many time steps, but also the usage of special numerical schemes without dispersion in the longitudinal direction $[16,17]$ to avoid an accumulation of errors. For the example of the rectangular collimator the CPU times for the direct and the indirect methods are given in Table I. The result for Eq. (18) was obtained with 50 modes calculated in advance. Equation (21) was solved with a conjugate gradient method. The direct integration was done along a distance of $1 \mathrm{~m}$ in the outgoing rectangular waveguide with the numerical scheme for Maxwell's equations described in [17].

The new indirect method was also used for the accurate calculation of collimator wakefields [22]. It was interesting to observe that the kick factor [1] depends strongly on the length of the interior collimator pipe, the effect not described in the literature so far. Note that this problem was very difficult to treat in three dimensions satisfactorily with the old techniques.

\section{CONCLUSION}

In this paper we extended available techniques and introduced a new general method for indirect integration of the wake potential in three dimensions. The algorithms developed are checked numerically and their efficiency is 
confirmed by the solution of real accelerator problems $[20-22,24]$.

\section{ACKNOWLEDGMENTS}

I would like to thank M. Dohlus, K. L. F. Bane, A. Kling, T. Limberg, and N. Watson for useful discussions and corrections. This work was supported by the EUROFEL project.

[1] A. W. Chao, Physics of Collective Beam Instabilities in High Energy Accelerators (John Wiley \& Sons, New York, 1993).

[2] B. W. Zotter and S. A. Kheifets, Impedances and Wakes in High-Energy Particle Accelerators (World Scientific, London, 1998).

[3] G. Aharonian, R. Meller, and R.H. Siemann, Nucl. Instrum. Methods 212, 23 (1983).

[4] T. Weiland, Nucl. Instrum. Methods 216, 31 (1983).

[5] R. L. Gluckstern and F. Neri, IEEE Trans. Nucl. Sci. 32, 2403 (1985).

[6] O. Napoly, Part. Accel. 36, 15 (1991).

[7] O. Napoly, Y. Chin, and B. Zotter, Nucl. Instrum. Methods Phys. Res., Sect. A 334, 255 (1993).

[8] T. Weiland and R. Wanzenberg, DESY Report No. DESY M-91-06, 1991.

[9] S. A. Heifets, SLAC Report No. SLAC/AP-79, 1990.

[10] V.E. Balakin, I. A. Koop, A. V. Novokhatskii, A. N.
Skrinskii, and V. P. Smirnov, in Proceedings of the 6th AllUnion Conference on Charged-Particle Accelerators, USSR, 1978.

[11] T. Weiland, in Proceedings of the 11th International Conference On High-Energy Accelerators, Geneva, Switzerland, 1980.

[12] T. Weiland, IEEE Trans. Nucl. Sci. 30, 2489 (1983).

[13] Y. H. Chin, CERN Report No. CERN-SL-94-02-AP, 1994.

[14] MAFIA Collaboration, MAFIA manual, CST GmbH, Darmstadt, 1997.

[15] I. Zagorodnov, R. Schuhmann, and T. Weiland, J. Comput. Phys. 191, 525 (2003).

[16] S. Novokhatski, SLAC Report No. SLAC-PUB-11556, 2005.

[17] I. Zagorodnov and T. Weiland, Phys. Rev. ST Accel. Beams 8, 042001 (2005).

[18] W. K. H. Panofsky and W. A. Wenzel, Rev. Sci. Instrum. 27, 967 (1956).

[19] R.E. Collin, Foundations for Microwave Engineering (McGraw-Hill, Inc., New York, 1992).

[20] K.L.F. Bane and I. A. Zagorodnov, SLAC Report No. SLAC-PUB-113888, 2005.

[21] M. Dohlus, T. Limberg, and I. Zagorodnov, DESY Report No. TESLA-FEL 2005-10, 2005.

[22] I. Zagorodnov and K. L.F. Bane, in Proceedings of EPAC 2006, Edinburgh, Scotland (THPCH036, 2006).

[23] H. Henke and W. Bruns, in Proceedings of EPAC 2006, Edinburgh, Scotland (WEPCH110, 2006).

[24] I. Zagorodnov, T. Weiland, and K.L.F. Bane, in Proceedings of PAC 2003, Portland, OR, 2003. 